\title{
Understanding Pointing Problems in Real World Computing Environments
}

\author{
Amy Hurst Jennifer Mankoff Scott E. Hudson \\ Human Computer Interaction Institute, Carnegie Mellon \\ 5000 Forbes Ave, Pittsburgh, PA 15213 \\ \{akhurst, jmankoff, scott.hudson\}@cs.cmu.edu
}

\begin{abstract}
Understanding how pointing performance varies in real world computer use and over time can provide valuable insight about how systems should accommodate changes in pointing behavior. Unfortunately, pointing data from individuals with pointing problems is rarely studied during real world use. Instead, it is most frequently evaluated in a laboratory where it is easier to collect and evaluate data. We developed a technique to collect and analyze real world pointing performance which we used to investigate the variance in performance of six individuals with a range of pointing abilities. Features of pointing performance we analyzed include metrics such as movement trajectories, clicking, and double clicking. These individuals exhibited high variance during both supervised and unsupervised (or real world) computer use across multiple login sessions. The high variance found within each participant highlights the potential inaccuracy of judging performance based on a single laboratory session.
\end{abstract}

\section{Categories and Subject Descriptors}

H.5.m [Information Interfaces and Presentation]: User Interfaces input devices and strategies

\section{General Terms}

Measurement, Performance, Human Factors.

\section{Keywords}

Pointing performance, motor impairments, data collection in the wild

\section{INTRODUCTION}

Computer technology has become an integral component in many people's lives for social, educational, entertainment and professional applications. However, computers are not universally accessible, and there is a growing population of individuals who are motivated to use a computer but find it physically difficult to do so. One reason computers can be difficult to use is that they do not adapt to a user's changing performance. While technology exists to improve pointing performance, many users either rarely configure it, or do not know how to configure it. Currently one of the best ways to learn the optimal configuration of this technology is through an assessment by a computer access specialist. Unfortunately, this solution is

\section{Fill in with ACM Copyright information after submission}

frequently imperfect as it is usually done in a laboratory during one visit. Studying pointing performance in real world use offers a more complex view of 1) how pointing performance varies over time within individuals, 2) how performance varies across individuals, 3) how performance varies across different pointing tasks, and 4) the limitations of laboratory data.

Performance data is frequently collected in the laboratory by instructing participants to perform repetitive pointing tasks and measuring the movement and accuracy of each trial. While this technique is one valuable way to collect data, it can miss several "real world" problems that an individual can encounter when using a computer on their own or in the wild. However, collecting real world data can be difficult because it is not as controlled as data from a laboratory study. This leads to problems including not knowing the user's intent and having to work across arbitrary (and potentially unknown or unique) applications.

We developed software and analysis tools to evaluate real world pointing performance. We used our tools to collect pointing data from six individuals with a range of disabilities who used our computers between 2 and 120 sessions. We compare their pointing performance on a controlled pointing task to their pointing performance during real world use. This comparison helps illustrate how much an individual's performance can vary, and the risk of configuring accessibility software on data from one pointing session. We also analyze the applications each user chose to interact with and evaluate their performance.

We first describe real world pointing errors found in other researchers' laboratory studies. Next we describe our performance metrics of both single and double clicks and how we analyze slips, movement trajectory, and button interaction, in Section 3. Our techniques to collect real world data are described in Section 4. Section 5 describes how we found high variability in performance within users over a series of sessions of real world use. Our results suggest the need for multiple assessments of individuals with pointing problems, ideally based on real world and laboratory pointing data.

\section{REAL WORLD POINTING ERRORS}

Unlike laboratory evaluations of pointing behavior, real world data lacks information about user intent. In a laboratory study, the participant is given a very specific task, such as "move the cursor to the box and click" and is scored on how long it took them to do that task and how accurate they were. However, real world computer use can be much more ambiguous as there is seldom only one possible correct action. One example is when the cognitive components of interaction, which are usually controlled for in a laboratory study, further confuse the data (such as deciding at the last minute that a button should not be pressed). With real world data the experimenter must divine (1) what action was completed and (2) whether that was the user's intent. Only 


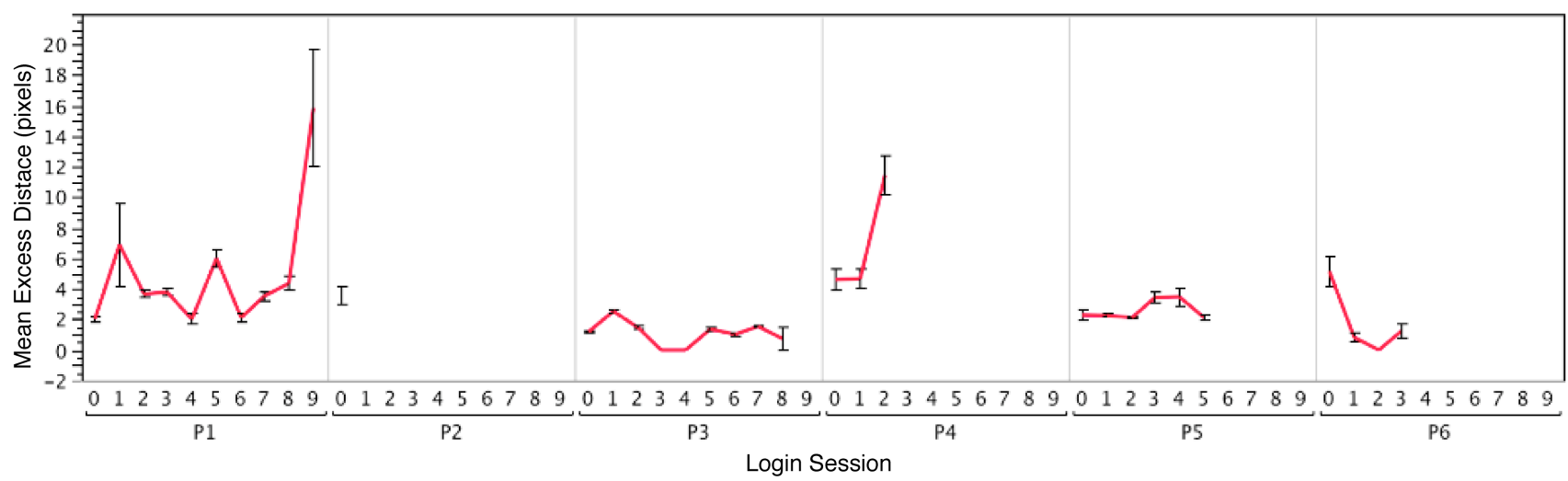

Figure 1. Comparison of the mean excess distance traveled by each user across login sessions. Login 0 was from a baseline clicking task, all other data is from double clicking a desktop icon or one of the clicking games.

then can the experimenter reasonably label data as erroneous. For example, if a user is surfing the web and clicks on a link and then immediately presses the "back" button, it is not clear if he or she accidentally clicked on that link or if the new web page simply was not interesting.

One way of handling this ambiguity is to focus on lower-level events. For this analysis, we focus on targeting events in which the user moves the mouse over a target and then clicks or double clicks on it. These types of motions have the advantage that (1) a large subset of them are easily segmented from other pointing data since they typically start with a still mouse and end with a click that results in a visible change on screen (2) they have been studied extensively in the laboratory. For this reason, our measures focus on errors that occur during clicking (or double clicking); and pointer motion characteristics that describe the motion to the target.

Detailed characteristics of a user's motion can also be indicative of pointing problems. We next present several characteristics of pointer interaction hat can be seen as likely indicators of difficulty.

\subsection{Clicking and Double Clicking}

Laboratory studies indicate that clicking and double clicking errors tend to fall into four major categories.

- Too many buttons: This occurs when a user accidentally presses both pointer buttons at the same time. In a laboratory study Trewin [10] observed 13 of 27 incorrect button presses where both buttons were pressed with no adaptation software.

- Accidental click: A user may click when he or she did not intend to. For example, a user may click partway through a mouse motion to a target, or may click the mouse button without moving the pointer [9]. Some laboratory studies may classify this type of error as missing a target, or do not measure it at all.

- Double click speed: Double clicking is a common task in modern GUIs, especially when interacting with items on a desktop. Double clicking is more difficult than single clicking for individuals with pointing problems [9]. For example, in a laboratory study of multiple clicking Trewin found a $39.5 \%$ error rate from motor impaired users performing two double clicks and six triple clicks, and a median error rate of $28.3 \%$ for single clicks. Able bodied users had a $9 \%$ error rate on multiple clicks, and a median error rate of $6.3 \%$ for single clicks. These errors were the result of positioning errors, moving during a click, or long delays between clicks. In this study, the delay between clicks in the motor impaired group ranged from $100 \mathrm{~ms}$ to $333 \mathrm{~ms}$. Click times for the able bodied group were between $50 \mathrm{~ms}$ and $100 \mathrm{~ms}$.

- $\quad$ Slips: Slipping can occur during either single or double when the user presses the mouse button, involuntarily moves the mouse off the target, and then releases the mouse button[10].

\subsection{Motion Characteristics}

To assess pointer motion, we use two performance measures that are target agnostic: direction change and excess distance traveled.

- Direction changes: Mackenzie defines Movement Direction Change (MDC) as the number of times the pointer path changes direction relative to the task axis and Orthogonal Direction Change (ODC) as the number of times the change in pointer path is orthogonal to the task axis. [6]. In our analysis we simplify this as the number of X or Y direction changes, illustrated in figures 7 and 8 .

- Excess distance traveled: Keates defines this as the ratio of the actual distance traveled relative to the minimum straight line distance [4]. This ratio is 1 when the cursor moves in an efficient, straight line from the source point to the target and higher when the cursor wanders. Figure 1 shows how the average score of participants in our study changed over time on this measure.

\section{DETECTING POINTING ERRORS}

We developed software to collect pointing, keyboard and visual data from any Windows application. This software extends DART (Disruption and Recovery Tracker) [3] a suite of system monitoring components that run in the background to $\log$ pointing, keyboard, and window events. Our software takes a 200x200 pixel screen capture (centered on the cursor) immediately before a button press, and during it. This code is written in $\mathrm{C}++$ and $\mathrm{C \#}$. Data were segregated into sessions by a login system written in Visual Basic that controlled all logging 
software. Log file parsing was writing in PERL and the difference image techniques are written in JAVA ${ }^{\text {tm }}$.

The following subsections describe how we calculated the measures in the previous section. While not an inclusive set of all possible measures, such as analysis of the targets, the set of measures we chose allows us to perform this initial survey of real world pointing behavior. All measures were calculated between pairs of clicks. For this analysis, a motion started at the end of one click and ended at the beginning of the next click.

\subsection{Clicking Problems}

\subsubsection{Too Many Buttons}

There is no situation in which both the left and right mouse buttons should be pressed in the applications our participants used. We are able to determine how many buttons on the mouse were pressed through log file analysis. We check for the occurrence of multiple button presses (left or right button) after a button press and check if the other is still pressed.

\subsubsection{Accidental Clicks}

There are two situations in which a user may click repeatedly without effect. The first is an attempted, but failed click or double click. The second is an accidental click. Most operating systems nearly all applications, including the ones our participants used, give graphical feedback for all successful clicks. So, for example, the user may click in the empty desktop space near a desktop icon (an accidental click) and then click on the icon itself (changing the target of the click). On the other hand if the user clicks on a desktop icon, pauses, and clicks again, leading to a failed double click, he or she is clicking on the same target. In both examples, the screen image does not change. However, if the user double clicks successfully, an application will open, changing the screen image.

We detect accidental clicks with a hybrid approach that automatically analyzes both the detailed input event log file and the images captured during the button down and up events. To see if a pair of clicks meet the criteria for an accidental click, we compare the images our software captures that are centered on the target at the button down event of both clicks. First, we align the pair of images by the distance between the cursor locations at both events to account for small differences due to slight movements of the cursor during a click. Next, we calculate the difference image, literally a subtraction of the two images, and if the images are not different, we label the first click as an accidental click. One small limitation of this technique is that we can only realign images if the distance moved between the clicks is smaller than our captured image width.

\subsubsection{Double Click Speed}

We define double click speed as the time between two consecutive clicks that took place on the same target. Target comparisons are done using difference imaging, as with accidental clicks.

\subsubsection{Movement During a Single Click (Slipping)}

We define a slip as any distance a pointing device moves while a pointer button is pressed. For this data, we only analyze applications that do not include drags. To this end, we also hand coded and removed all drags due to menu use. For each click, we calculate the "slip" as the Euclidean distance between button up and down events.

\subsection{Pointing Motion Characteristics}

\subsubsection{Direction Changes}

For our analysis, rather than calculating the task axis and the amount of movement direction change/orthogonal direction change, we focused on the simpler measures of number of $\mathrm{X}$ direction changes and $\mathrm{Y}$ direction changes from one click to the next.

\subsubsection{Excess Distance Traveled}

We calculate the ratio of the total distance traveled and the minimal Euclidean distance between each pair of clicks. This ratio represents the efficiency of the trajectory; it is 1 when the cursor moved in a straight line from the source point to the target, and longer otherwise. One limitation to analyzing cursor trajectories in real world data is that we cannot determine if path data is a long or curvy movement made because the user was having a pointing problem or rather they changed their mind during the movement and selected a different target than their initial intent.

\section{DATA COLLECTION}

\subsection{Method}

Two computers were installed in a popular, pre-existing computer laboratory at Pittsburgh's United Cerebral Palsy Center (UCP). Participants in the study were allowed to use these computers during their free time, which was typically before the morning classes started, during lunch, and after classes. Since participants were free to work at any pace they chose, they could also take breaks and stop anytime they wanted, without explanation to an experimenter.

Baseline performance data was collected using the IDA [5] software suite, a software suite developed to support clinical evaluation of problems with keyboard and pointer use. This software is designed for use by clinicians as part of their assessment process for determining assistive technology needs. Participants performed 30 trials of the AIM task, a standard task included with IDA. In the IDA software, participants were given two minutes to move the cursor to a blue square and click the left button. The software did not alert the user if they make an error, and let them work on the task until they reach the two-minute time limit. IDA automatically varies the size and location of the targets throughout all of the tasks. The mouse gain during the baseline trials was set to the default value (10) and Enhanced Pointer Precision was turned on.

Participants completed the study in two phases. After giving informed consent to the study, participants began the first phase where they completed baseline clicking tasks using IDA and answered demographic questions. This phase lasted 1-2 hours and participants were compensated \$25 for completing it. Participants were then assigned a unique participant number which they could use to log into the study machines at any time.

Due to external constraints, our computers differed from those already in the laboratory in that they were not networked and were only used by participants in the study. They also had the same productivity tools as the other computers in the laboratory, but different games. Participants could use the study machines as they pleased, however, we did require them to use the USB mouse and keyboard provided by DELL with the machines. Participants were permitted to change the pointing settings (such as mouse acceleration) on the computers, however none chose to do so. 
Participants were told, and reminded with a sign, that all their data on these computers would be recorded, and they should ask the experimenter if they would like any of their sessions to be removed. The experimenter visited the center one or two times a week to collect data, and answer questions about the computers.

To motivate users to use the study computers, a raffle was held every month for participants who logged into the machines at least once during that time period. Raffle winners received \$25.

\subsection{Participants}

Using our described method, we collected real world data from 11 participants starting in January 2008, and data collection is still ongoing (as of publication date). In this paper, we present a subset of our total dataset from 6 participants (4 female) who used the computers at least twice in a four-month period from $1 / 08$ to $5 / 08$. The mean age of our participants was 40.5 years (SD $=7.77$ ) and 2 of them used a mouse with their left hand. Activity and frequency of use varied widely across participants, but ranged from 2 sessions to 120 sessions of length 2 minutes to 74 minutes. For participants who logged many sessions, we randomly selected 9 for the analysis described next.

When asked about experience configuring accessibility settings, participants P3 and P5 were the only participants who said they had ever made adjustments to the pointing settings (both liked to increase the pointer gain). Participants P3 and P4 both said they like to use the Sticky Keys feature to help with typing. Overall participants did not know all of the available configuration options available to them, and did not know details about the best settings for them. One participant even remarked that he was a little scared to change these settings because he was concerned he would not be able to set them back.

P1. This participant has been using computers for about 5 years, and uses them 11 to 20 hours per week. He learned how to use a computer through classes at the center and uses a standard keyboard and mouse. He frequently uses computers to play cards, puzzle games and also enjoys using the Internet to look up information. P1 is paralyzed from the waist down and has memory and motor impairments (including an intermittent tremor).

P2. This participant has been using computers for more than 10 years and uses them 2-5 hours per week. He trained himself to use a computer and uses a standard computer and mouse. He frequently uses a computer for email, getting information and writing reports. He has a memory impairment, which can make navigation within an application difficult.

P3. This participant has been using computers for more than 10 years, and on average uses them 6 to 10 hours a week. She learned how to use a computer through college and high school classes. She uses a standard mouse with a desktop computer and a touchpad with a laptop (which she says she can use just as well as a mouse). She frequently uses a computer for email, writing reports, and playing card and puzzle games. This participant has cerebral palsy, upper extremity impairment, and a minor vision impairment.

P4. This participant has been using computers for more than 10 years, and uses them for 6-10 hours per week, and learned how to use them on her own. For the last five years she has been using a touchpad to interact with a computer at home. She is able to use a standard mouse, but her limited dexterity (due to an upper extremity impairment and arthritis) can make mouse clicking and navigation difficult. She frequently uses computers for email, pinball and card games, and writing stories.

P5. This participant has been using computers for more than 10 years, and on average uses them 6 to 10 hours a week. She learned how to use a computer through classes in school. She traditionally uses a standard keyboard and mouse. She briefly used a voice activated system, but stopped using it because it was too sensitive to her abilities as they changed day to day, and it ultimately slower than using a keyboard and mouse. She frequently uses a computer for email, getting information, conducting business and writing reports. She has cerebral palsy and upper extremity impairment.

P6. This participant has been using computers for about 3 years and uses them about 6 to 10 hours a week. She learned how to use a computer through classes at UCP, and uses a standard mouse and keyboard. She frequently uses a computer for email, getting information, and writing. She has a memory and cognitive impairment and has difficulty staying focused.

\section{RESULTS}

Our participants used these computers for a variety of tasks including playing clicking games and card games, solving jigsaw puzzles, making greeting cards and typing documents. Overall, the majority of the data we collected was from clicking games, specifically a matching game called the "Same Game", and a memory game called "Memory Blocks". While Microsoft Word was the third most popular application, we collected eight times more data from the two clicking games than Microsoft Word. Participants P2 and P6 did not use the computers as frequently as the other participants and did not play either popular clicking game. They are included in this analysis for their interactions with the desktop, and P2s overlapping button press problem while using Microsoft word.

All participants launched each application by opening a desktop icon (none of them used the start menu). In this paper we focus most of our analysis on participants' real world performance on the three common tasks for which we have the most data: launching applications by double clicking on desktop icons, and two clicking games (Same Game and Memory Blocks).

The characteristics of certain applications make a subset of clicking movements more straightforward to interpret. This is the approach used in our analysis. For example, we know that the user should not drag the cursor while playing a game that only responds to clicks. We may also consider the chain of events leading up to actions in our analysis. For example, multiple interactions with the same application icon on the desktop may imply a failed double click attempt.

\subsection{Double Clicking Errors}

\subsubsection{Analysis notes}

Analysis of double clicking a desktop icon is relatively straightforward because the Windows Operating System enforces the following requirements.

1. Desktop icons are activated only after they are clicked twice in succession.

2. The speed between these clicks is critical: if the time between the clicks is longer than the set Double Click Time, the action is not registered as a double click. 


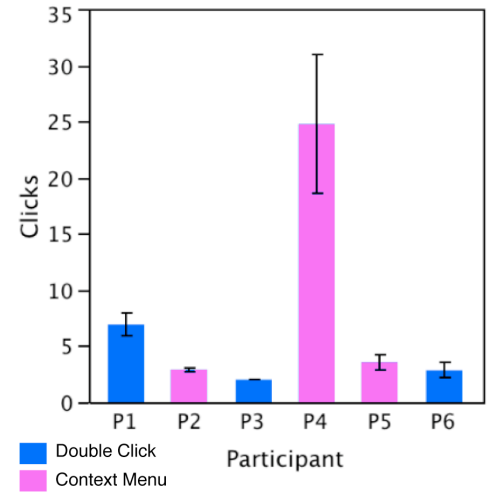

Figure 2. Mean number of clicks it took each participant to activate a desktop icon.

3. Note that an individual can right click the desktop icon and open the application through a context menu. Three of our participants chose to do this exclusively while the other three relied exclusively on double clicking to open applications. Our analysis only includes the latter three participants.

\subsubsection{Double Clicking Performance}

We saw a wide range in the number of clicks performed to open a desktop icon, which ranged from 2-20 when double clicking (Figure 2). Participants P1, P3 and P6 had a mean double click speed of $1315.85 \mathrm{~ms}(\mathrm{SD}=303.88)$. This is more than twice the Windows limit of 500ms [7], beyond which Windows interprets two consecutive clicks as consecutive single clicks rather than a double click event. Participant P1's mean time was $1090.25 \mathrm{~ms}$ $(\mathrm{SD}=194.07 \mathrm{~ms})$, P3's mean time was $2616.50 \mathrm{~ms}$ (SD $=$ $544.22 \mathrm{~ms}$ ) and P6's mean time was 1966.75 (SD $=638.15 \mathrm{~ms})$. Performance varied so much that the standard deviation in time between double clicks was larger than the default double click speed for participants P1 and P3. Note that participant P3 had the lowest number of average clicks to open the icon (illustrated in Figure 2) yet the highest mean time to open the item. We think the long time between clicks is attributed to this user taking breaks or waiting to see if their action opened the item. Figure 3 illustrates how these times varied across login sessions. Note that the baseline session is not included here because it did not include a double clicking task.

\subsection{Clicking Errors And Pointer Motion}

Of all the software available to our participants two clicking games, The Same Game and Memory Blocks, were the most

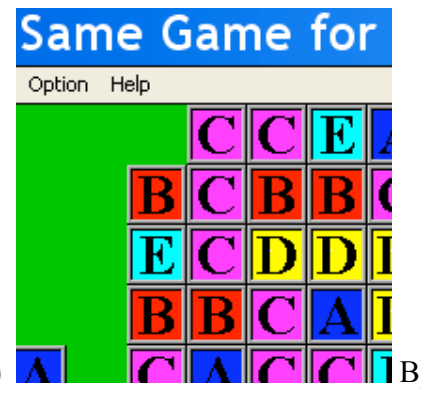

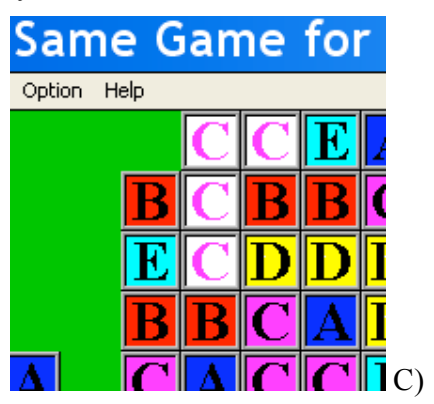

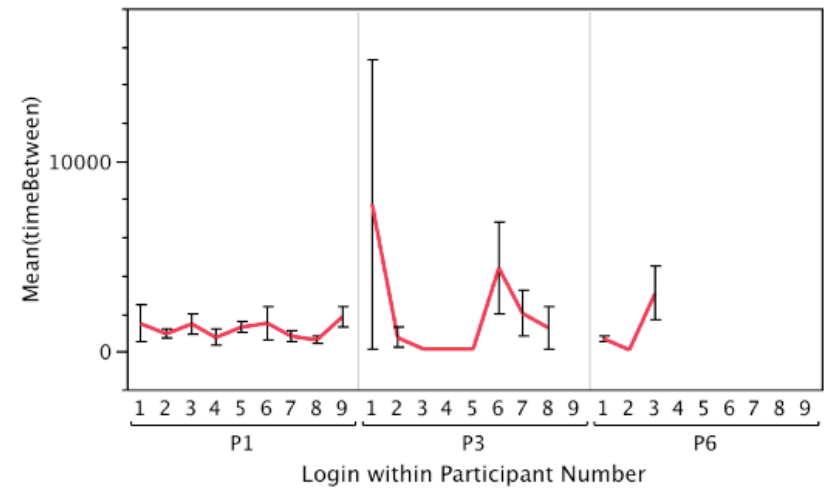

Figure 3. Graph of the time between double click attempts by participant across login sessions.

popular applications with participants $\mathrm{P} 1, \mathrm{P} 3, \mathrm{P} 4$, and $\mathrm{P} 5$. These two games are relatively simple and only require the use to single click on a target so they are a great way to understand clicking errors and pointer motion. We focus our analysis of single clicking on these two clicking games since they had simple interaction paradigms, and we had a lot of data from them. Figure 4 shows examples of the interaction of these games.

\subsubsection{Analysis Notes}

The most commonly used application was the Same Game. This is a game where participants click on connected letterboxes with matching letter and color. This game was so popular that one of our participants upgraded our copy of this game with a version she owned.

Analysis of performance of the Same Game is simplified by the game's interaction requirements.

1. Right clicks are ignored.

2. Participant must click on set of connected letterboxes twice to make them disappear. The speed of these clicks does not matter.

3. Once the participant has clicked on a letterbox, another click on anything else is an error, and the selection is lost.

4. All letterboxes are 30x31 pixels, but the clickable targets grow as the letterboxes are connected.

The memory game was the second most popular application with our participants, and is similar to the Same Game in that it also only requires the user to click on square targets. In this game
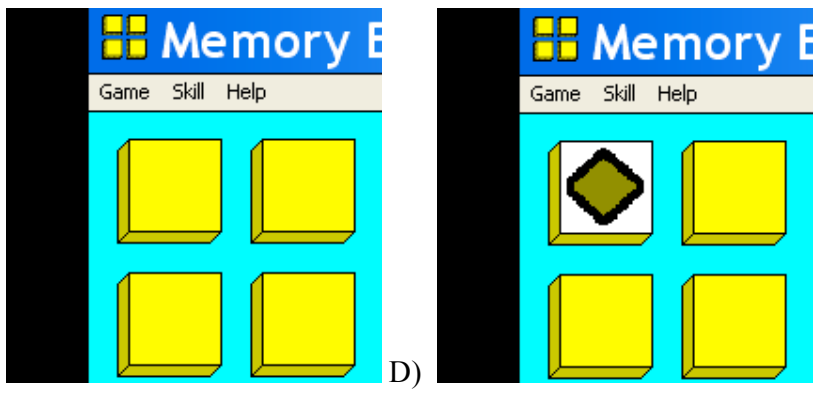

Figure 4. Screenshots from the two most popular games A \& B) Same Game, where participants need to click on a connected group of blocks with matching colors and letters. B shows the currently selection of connected blocks. If user clicks on selected area, all highlighted blocks will disappear. C \& D) Screenshots of the Memory Blocks Game where participants click on blocks to flip them over and match blocks with identical icons. This is a memory game because tiles are flipped back over after two have been viewed, so participants must remember the location of the icons. 


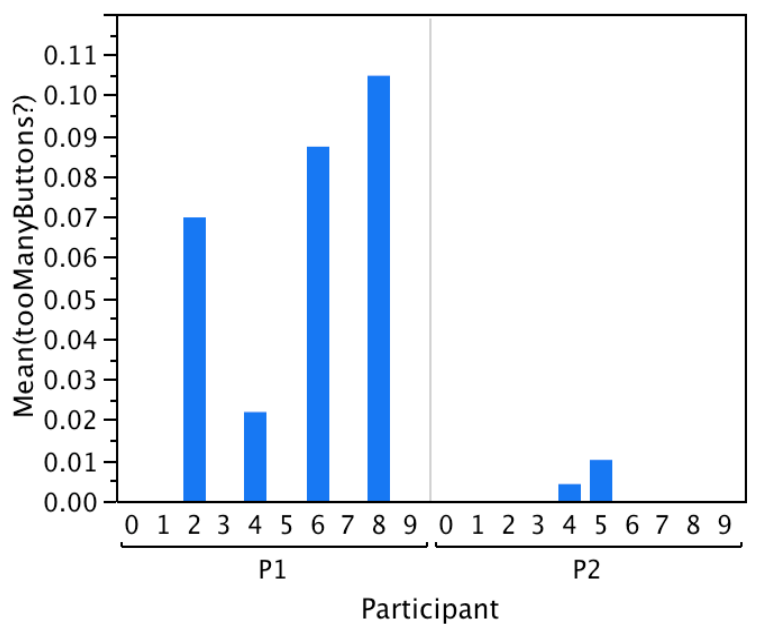

Figure 5. Plot of number of clicks with too many buttons across all logins and sessions. Login 0 is from the baseline task.

participants click on targets to flip blocks, and continue to select targets until they have found two that match. When two matching blocks have been found, they both disappear. This game continues until all blocks have been matched.

Analysis of performance of the Memory Game is simplified by the game's interaction requirements.

1. Right clicks are ignored.

2. Clicks on anything other than a block are ignored.

3. Only two blocks are flipped at any given time.

4. All blocks are 50x50 pixels.

As a result of these interaction models, we were able to simplify the detection of accidental clicks: Since the user would never intend to double click, we could simply look for clicks that did not change anything on screen.

\subsubsection{Too Many Buttons}

Participants P1 and P2 were the only participants who had overlapping button presses from pressing multiple buttons during a click. Figure 5 illustrates the frequency of this performance problem across 9 login sessions for all applications. In this figure, P1 only exhibited this problem during sessions 2,4,6 and 8 . Participant P2 had three overlapping button presses, which happened while using Microsoft Word during sessions 4 and 5.

\subsubsection{Slips}

Recall that a slip is defined by the movement of the pointer while still pressing the mouse button during a click. Figure 6 shows the average value for this measure in each session that was analyzed for each participant. The $0^{\text {th }}$ session shown for each participant is the baseline session, and the following are from real world use. In Figure 6, there is data from 10 sessions from $\mathrm{P} 1,1$ from $\mathrm{P} 2$, and so on.

In Figure 6, the mean slip distance varied from 0 pixels to over 20 pixels, the mean slip distance for all participants across all sections was 5.9 pixels $(\mathrm{SD}=19.45)$ Participants $\mathrm{P} 4$ and P5 had the most problem with slips $\{\mathrm{P} 4$ mean $=21.34$ pixels, $\mathrm{SD}=43.96$; P5 mean $=10.25$ pixels, SD $=24.49\}$. Large slips can cause a click to fail because the pointer moves off the selected interactor before it is released.

\subsubsection{Accidental Clicks}

All successful clicks in the Same Game and Memory Blocks games result in a visual change. $17.5 \%$ of the clicks we collected in these games resulted in no visual change, and were counted as accidental clicks (errors). The percentage of clicks that were accidental in the two clicking games for all participants who played these games $\{\mathrm{P} 1=19.2 \%, \mathrm{P}=13.9 \%, \mathrm{P} 4=29.4 \%$, P5 $=17.7 \%$. Note: Participants P2 and P6 did not play either clicking game. A high percentage $(66.72 \%)$ of these accidental clicks involved a slip during the click. Since we do not know which target the user was trying to click on, we do not know which of the clicks with slips were failed target selections or not.

\subsubsection{Direction Changes}

Figures 7 and 8 illustrate the average number of direction changes per motion along the $\mathrm{X}$ and $\mathrm{Y}$-axis. As with Figure 6, session 0 is the baseline session. Participants P1, P4 and P5 experienced high numbers of direction changes most frequently, with means ranging from more than 30 to 0 across different sessions. Total scores for these individuals across all sessions for $\mathrm{X}$ direction changes were $\{\mathrm{P} 1$ Mean $=4.8, \mathrm{SD}=8.62 ; \mathrm{P} 4$ Mean $=2.8, \mathrm{SD}=$ 7.45; $\mathrm{P} 5$ Mean $=3.17, \mathrm{SD}=5.14\}$ and $\mathrm{Y}$ direction change were $\{\mathrm{P} 1$ Mean $=2.14, \mathrm{SD}=3.13 ; \mathrm{P} 4$ Mean $=3.11, \mathrm{SD}=7.51 ; \mathrm{P} 5$ Mean $=3.63, \mathrm{SD}=7.47\}$.

\subsubsection{Excess Distance Traveled}

Figure 1 illustrates the ratio of movement length to minimal movement length is shown. When this value is close to 1 , the user moved in a straight line from the source to the target. As with Figures 6,7 and 8 , session 0 is the baseline session.

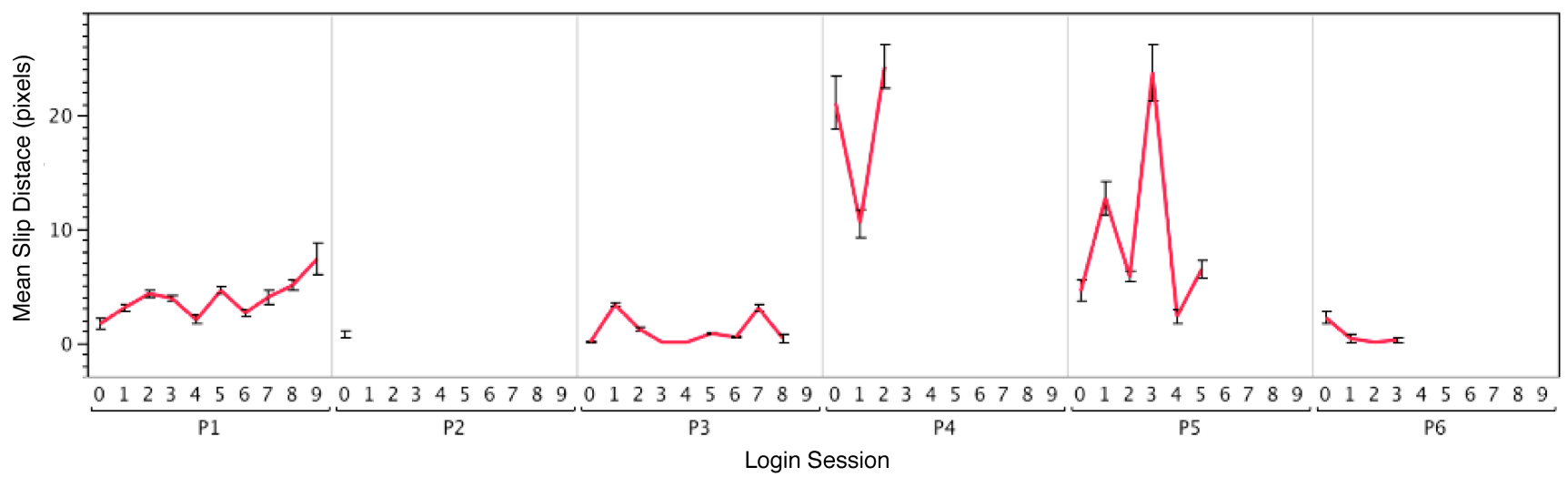

Figure 6. Comparison of the difference between the mean distance slipped by each user across login sessions. Login 0 was from a baseline clicking task, all other data is from double clicking a desktop icon or one of the clicking games. 


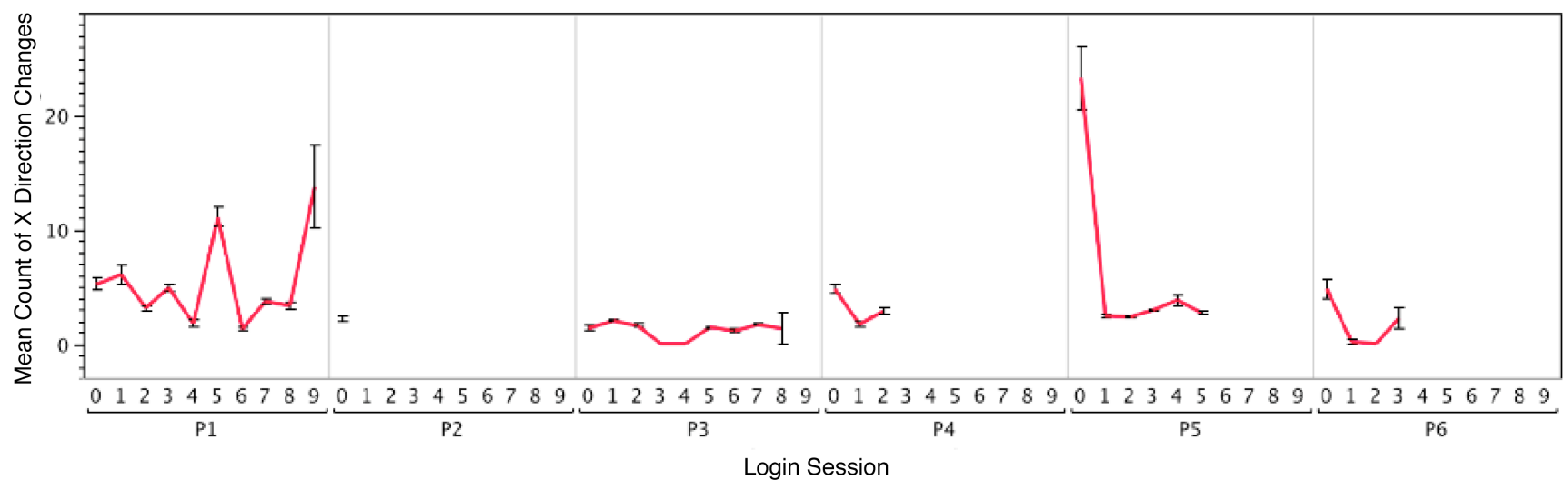

Figure 7. Comparison of the difference between the mean number of direction changes in the $X$ direction by each user across login sessions. Login 0 was from a baseline clicking task, all other data is from double clicking a desktop icon or one of the clicking games.

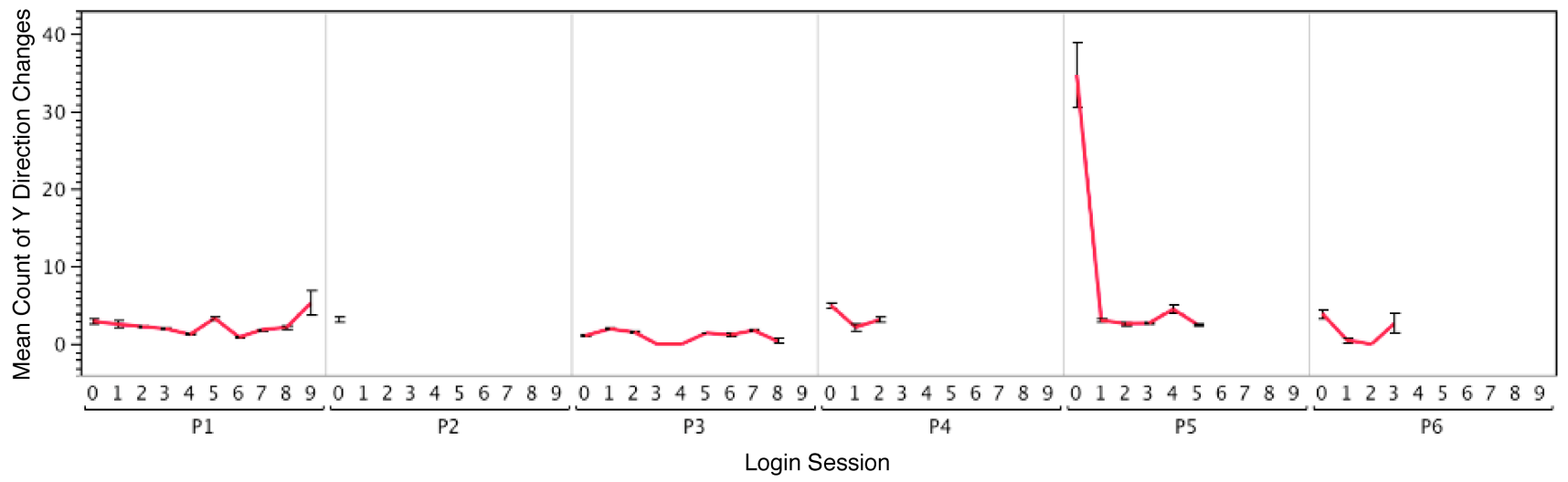

Figure 8. Comparison of the difference between the mean number of direction changes in the $Y$ direction by each user across login sessions. Login 0 was from a baseline clicking task, all other data is from double clicking a desktop icon or one of the clicking games.

P1's mean excess distance traveled ratio is low (below 2.02-2.11) in the baseline session and real world sessions 4 and 6 . However P1's performance on this metric rises as high as 6.9 in session 1 and 15.86 in session 9 .

\subsection{Analysis of Variance Across Sessions}

As seen in Figures 1,6,7,8 there was high variability across and within sessions. We tested for homogeneity of variance, using Levene's test, which found unequal variance across all measures $(\mathrm{p}<.001)$ across sessions and across participants $(\mathrm{p}<0.001)$.

The jagged up and down scores on metrics from one session to the next, visible in all figures, illustrates the high variance that occurred across sessions. Participants experienced an unpredictably wide range of performance across sessions. This is visible in our data: there are several instances where performance during baseline collection (done as a laboratory study) differed dramatically from real world use. For example, P1 did not have a problem with overlapping button presses during the baseline task, but did several times during real world sessions. As expected, there was no significant correlation between login session and performance for any the participant across any of the measures.
In addition to high variance across sessions, there was also high variation within sessions, as evidenced by the wide error bars, especially prominent on sessions with worse performance. For example, participants P4 and P1 had the highest variance on the measure of excess distance traveled (Figure 1) across sessions (SD for P1 $=10.22$; SD for P4 $=27.8$ ), and they also had the highest scores (as high as 15.86 in session 9 for P1). In contrast, the standard deviation on this measure for the other participants was between 3.22 and 5.87, and their scores were also generally low. A similar pattern is visible in Figures 6, 7 and 8.

Variance was correlated among three of our measures, providing evidence that in bad sessions, users experienced multiple types of difficulties. For a given participant, a cross correlation showed that the variance of distance slipped is highly correlated with the variance of the number of changes in the $\mathrm{Y}$ direction $(\mathrm{r}=.90, \mathrm{p}<$ $.05)$ and the variance of the distance moved/distance between targets $(r=.82, p<.05)$. Other pairings of our measures were not significantly correlated. 


\section{DISCUSSION AND FUTURE WORK}

Our results characterize performance on a set of 6 different measures over time. While we do not yet have sufficient data to accurately describe performance for large classes of users, we show that an individual's pointing performance can vary within a session as well as across sessions. This variability is significant, resulting in some sessions where errors are frequent and others where errors are not, and this variability is visible even on the scores of our most impaired participants.

Based on our findings in this paper, we feel that real world data collection is a valuable method to understand pointing performance with individuals whose performance can fluctuate due to medication, progression of a disease, or as a side effect of impairment. Additionally, unobserved and free form data collection techniques may free participants to take breaks and reduces some types of fatigue and stress present during more traditional laboratory studies.

Assessment of performance of laboratory data from one session from individuals with pointing problems is insufficient to see the variance of performance and may exclude or include errors not reflected in later sessions. However, laboratory data collection is important because it is a valuable way to be certain of the user's intent, and isolate certain variables.

Given current tools for analysis in the real world and the inherent limitations of constrained tasks in the laboratory, neither approach is currently able to provide a complete picture of an individual's pointing abilities. We feel that assessments should use both laboratory data and data about real world use to more accurately understand pointing ability.

In addition to exploring how to collect real world pointing data, this paper describes pointing performance variations from six individuals with cognitive and motor impairments. The variability in real world performance we observed provides further evidence that assistance software should assess and adapt to a user's current performance, rather than assume constant performance.

We are working to build software that will bridge the gap between an individual's current performance and the appropriate settings for an adaptation. In previous work, we developed several techniques to make sense of real world use including analyzing menu use to estimate expertise without a task model [1]. We also developed a classifier that could identify the appropriate assistive technology for a person based on laboratory data [2].

While our data was collected in a shared computer laboratory, this technique (and our software) could easily be used to gather the same kind of data on an individual's machine at home. As future work, we will continue to understand real world pointing performance and investigate how we can automatically detect these changes in real world use. Specifically we will build on our success in the laboratory to automatically detect pointing problems and predict the benefit of a particular adaptation and transfer that into the real world. As we are able to assess performance in real world computer use, we will start learning how we can best accommodate a user's current performance and match them to pointer adaptations to improve performance.

\section{ACKNOWLEDGMENTS}

We thank all our participants and the staff at United Cerebral Palsy Center in Pittsburgh for their time and commitment to help collect this data. This work was supported in part by the National
Science Foundation under grants EEC-0540865, IIS-0713509, IIS-0325351, IIS-0205644, the first author's NSF Graduate Student Research Fellowship; IBM Research; and the Pennsylvania Infrastructure Technology Alliance.

\section{REFERENCES}

[1] Hurst, A., Hudson, S. E., and Mankoff, J. 2007. Dynamic detection of novice vs. skilled use without a task model. In Proceedings of the SIGCHI Conference on Human Factors in Computing Systems (San Jose, California, USA). CHI '07. ACM Press, New York, NY, 271-280.

[2] Hurst, A., Hudson, S. E., Mankoff, J., Trewin, S. 2008. Automatically Detecting Pointing Performance. Intelligent User Interfaces. (Grand Canaria, Canary Islands, Spain). IUI 2008. ACM Press, New York, NY, 11-19.

[3] Iqbal, S. T. and Horvitz, E. 2007. Disruption and recovery of computing tasks: field study, analysis, and directions. In Proceedings of the SIGCHI Conference on Human Factors in Computing Systems (San Jose, California, USA). CHI 07. ACM Press, New York, NY, 677-686.

[4] Keates, S., Hwang, F., Langdon, P., Clarkson, P. J., and Robinson, P. 2002. Cursor measures for motion-impaired computer users. In Proceedings of the Fifth international ACM SIGACCESS Conference on Assistive Technologies (Edinburgh, Scotland). Assets '02. ACM Press, New York, NY, 135-142.

[5] Koester, H. H., LoPresti, E., and Simpson, R. C. 2005. Toward Goldilocks' pointing device: determining a "just right" gain setting for users with physical impairments. In Proceedings of the 7th international ACM SIGACCESS Conference on Computers and Accessibility (Baltimore, MD, USA). Assets '05. ACM Press, New York, NY, 84-89.

[6] MacKenzie, I. S., Kauppinen, T., and Silfverberg, M. 2001. Accuracy measures for evaluating computer pointing devices. In Proceedings of the SIGCHI Conference on Human Factors in Computing Systems (Seattle, Washington, United States). CHI '01. ACM Press, New York, NY, 9-16.

[7] Microsoft Developer Network, SetDoubleClickTime function, http://msdn.microsoft.com/enus/library/ms646263(VS.85).aspx (accessed 5/4/08)

[8] Paradise, J., Trewin, S., and Keates, S. (2005). Using Pointing Devices: Difficulties Encountered and Strategies Employed. In Proceedings of the 3rd International Conference on Universal Access in Human-Computer Interaction. (Las Vegas, Nevada, USA). UAHCI '05.

[9] Trewin, S. and Pain, H. (1999). Keyboard and mouse errors due to motor disabilities. International Journal of HumanComputer Studies 50(2), pp109-144.

[10] Trewin, S., Keates, S., and Moffatt, K. 2006. Developing steady clicks: a method of cursor assistance for people with motor impairments. In Proceedings of the 8th international ACM SIGACCESS Conference on Computers and Accessibility (Portland, Oregon, USA). Assets '06. ACM Press, New York, NY, 26-33. 
